\title{
Erratum: Zeeman-induced gapless superconductivity with a partial Fermi surface
} [Phys. Rev. B 97, 115139 (2018)]

Noah F. Q. Yuan and Liang Fu

(Q) (Received 29 June 2018; published 13 July 2018)

DOI: 10.1103/PhysRevB.98.039901

The second line of the pairing term $H_{P}$ above Eq. (3) is mistakenly written in the particle-hole channel. The correct second line should be written in the particle-particle channel as

$$
H_{P}=\Delta\left[i e^{-i \theta_{k}}\left(f_{\boldsymbol{k}}^{\dagger} f_{-\boldsymbol{k}}^{\dagger}-d_{\boldsymbol{k}}^{\dagger} d_{-\boldsymbol{k}}^{\dagger}\right)+\text { H.c. }\right] .
$$

In the rest of the original paper, the correct pairing term is used, such as Eq. (3), hence the calculations and results are not affected. 